\title{
Real-Time Evolution of the Electron Clouds of Transition Metal lons: Possible Electron-Pairing Medium in Unconventional High-Temperature Superconductors
}

\author{
Tiege Zhou ${ }^{1}$ (D) $\cdot$ Min Wang ${ }^{2} \cdot$ Xiaoguang Luo $^{1} \cdot$ Xu Zhang $^{1}$ \\ Received: 29 April 2019 / Accepted: 1 July 2019 /Published online: 5 July 2019 \\ (C) The Author(s) 2019
}

\begin{abstract}
The electron-pairing mechanism in unconventional high-temperature superconductors (HTS) has not been resolved. We proposed that the electron-pairing medium of unconventional HTS is the change of the electron clouds of transition metal ions, which is analogous to the lattice vibration in conventional superconductors. Real-time evolution of the electron clouds of transition metal ions under excitations in $\mathrm{La}_{2} \mathrm{Fe}_{2} \mathrm{As}_{2} \mathrm{O}_{2}$, FeSe sheet, and $\mathrm{HgBa}_{2} \mathrm{Ca}_{2} \mathrm{Cu}_{3} \mathrm{O}_{8}$ was calculated by the timedependent density functional theory (TDDFT). The characteristic frequencies are $160 \mathrm{meV}, 190 \mathrm{meV}$, and $250 \mathrm{meV}$, respectively. The frequencies are close to that of the lattice vibration in conventional HTS at high pressures, showing that the change of the electron clouds of the transition metal ions can be the electron-pairing medium.
\end{abstract}

Keywords Unconventional high-temperature superconductor · Time-dependent density functional theory · Electron-pairing medium

\section{Introduction}

The discovery of copper oxide $[1,2]$ and iron-based $[3,4]$ superconductors indicates that the electron-lattice interaction cannot explain the electron-pairing mechanism in unconventional HTS. According to the BCS theory $[5,6]$, the superconducting transition temperature $\left(T_{\mathrm{c}}\right)$ caused by the electron-lattice interaction (at normal pressures) cannot be higher than 40 K. In 1987, P. W. Anderson [7] has put forward the famous RVB model for copper-based superconductors. Other theories [8-10] have also been proposed. But P. W. Anderson [11] stated in 2007 that many theories about hightemperature superconducting electron-pairing mechanism might be in the wrong direction. Recently, orbital fluctuation

Tiege Zhou

zhoutg@nankai.edu.cn

1 College of Electronic Information and Optical Engineering, Nankai University, 300350 Tianjin, People's Republic of China

2 Hebei Advanced Thin Films Laboratory, Institute of Physics, Hebei Normal University, 050024 Shijiazhuang, People's Republic of China has been extensively studied [12-15], but the characteristic frequency of the fluctuation has been not obtained. The pairing mechanism for unconventional HTS is still under debate. There are two main viewpoints on the electron pairing. One is that there is a lattice-like medium. The other is that there is no lattice-like medium.

This paper explores the first point of view. We think that, if a medium does exist in unconventional HTS, the medium should change under excitations and the characteristic frequency should be close to that of the lattice vibration. Table 1 gives the maximum phonon frequencies and $T_{\mathrm{c}}$ of three typical conventional superconductors. It is reasonable to guess that the characteristic frequency of the electronpairing medium in unconventional HTS should be about $100-200 \mathrm{meV}$. If the frequency is too low or too high, it is unlikely to be an electron-pairing medium in HTS. If the frequency is too low, $T_{\mathrm{c}}$ cannot be so high. If the frequency is too high, it cannot be excited by free electrons.

Based on the above consideration, T. G. Zhou had studied eight typical unconventional superconductors $\left(\mathrm{Fe}_{2} \mathrm{KSe}_{2}\right.$, $\mathrm{La}_{2} \mathrm{Fe}_{2} \mathrm{As}_{2} \mathrm{O}_{2}, \mathrm{Nd}_{2} \mathrm{Fe}_{2} \mathrm{As}_{2} \mathrm{O}_{2}, \mathrm{Ba}_{2} \mathrm{Fe}_{4} \mathrm{As}_{4}, \mathrm{YBa}_{2} \mathrm{Cu}_{3} \mathrm{O}_{7}$, $\mathrm{HgBa}_{2} \mathrm{Ca}_{2} \mathrm{Cu}_{3} \mathrm{O}_{9}, \mathrm{Tl}_{2} \mathrm{Ba}_{2} \mathrm{CaCu}_{2} \mathrm{O}_{8}$, and $\mathrm{Bi}_{2} \mathrm{Sr}_{2} \mathrm{Ca}_{2} \mathrm{Cu}_{3} \mathrm{O}_{10}$ ) [18]. Under a static electric field, the electron clouds of transition metal ions change significantly. A pairing mechanism 
Table 1 Maximum phonon frequencies and $T_{\mathrm{c}}$ of three typical conventional superconductors

\begin{tabular}{llll}
\hline & $\mathrm{Pb}[16]$ & $\mathrm{MgB}_{2}[16]$ & $\mathrm{H}_{3} \mathrm{~S}[17]$ \\
\hline$\omega / \mathrm{meV}$ & 9 & 90 & 250 \\
$T_{\mathrm{c}} / \mathrm{K}$ & 7 & 39 & 164 \\
\hline
\end{tabular}

was proposed. When a free electron comes to a new place, the electron clouds of the neighboring transition metal ions will change. In this way, the charge densities around the free electron will decrease. When the free electron leaves, the electron clouds of the transition metal ions will not relax immediately, so that there will be a region lack of charge. Another free electron will be attracted. An attraction appears. This mechanism is essentially the same as the electron-lattice interaction, except that the medium is the change of the electron clouds, not the displacement of the ions.

Based on the previous studies, we have made a further investigation, investigating the frequencies of the change of the electron clouds of transition metal ions. The real-time evolution of charge densities under some excitation of $\mathrm{La}_{2} \mathrm{Fe}_{2} \mathrm{As}_{2} \mathrm{O}_{2}, \mathrm{FeSe}$ sheet, and $\mathrm{HgBa}_{2} \mathrm{Ca}_{2} \mathrm{Cu}_{3} \mathrm{O}_{8}$ has been calculated by TDDFT $[19,20]$ method. This paper will report the methods and results.

\section{Methods}

Calculations were conducted within the Octopus package [21, 22]. The generalized gradient approximation (GGA) of PerdewBurke-Ernzerhof (PBE) was used to describe the exchangecorrelation energy. The GGA+U method $[23,24]$ was considered to deal with the strong correlation of the $3 d$ electrons. HSCV pseudopotentials [25] were adopted. Approximated enforced time-reversal symmetry (AETRS) algorithm was used to approximate the evolution operator and the time step is $0.002 \hbar / \mathrm{eV}$.

\section{Results and Discussions}

\section{1 $\mathrm{La}_{2} \mathrm{Fe}_{2} \mathrm{As}_{2} \mathrm{O}_{2}$}

The $k$-point setting is $4 \times 4 \times 2$ and $5 p^{6} d^{1} 6 s^{2}$ of La, $3 p^{6} d^{6} 4 s^{2}$ of $\mathrm{Fe}, 3 \mathrm{~d}^{10} 4 \mathrm{~s}^{2} \mathrm{p}^{3}$ of As and $2 \mathrm{~s}^{2} \mathrm{p}^{4}$ of $\mathrm{O}$ are taken as valence electrons in the calculation. The real space spacing is $0.35 \mathrm{Bohr}$. The change of charge densities is induced by a timedependent electric field:

$f(t)=F_{0} \cos \left(\frac{\pi}{2} \frac{t-2 \tau_{0}-t_{0}}{\tau_{0}}\right) \cos \omega t$ If $\left|t-t_{0}\right|>\tau_{0}$, then $f(t)=0$.

where $F_{0}=1.0 \mathrm{eV} / \AA$, $\omega=1.8 \mathrm{eV}, \tau_{0}=5.0 / \mathrm{eV}$, and $t_{0}=5.0 / \mathrm{eV}$. The electric field is along the [111] direction. The excitation vanishes after 5000 steps and the charge densities vibrate freely thereafter. After 8000 steps, the charge densities are recorded. Figure 1 shows the crystal structure and the real-time evolution of the charge density. 100 represents the charge density difference between the 8100th step and the 8000th step and the same below. The change of the electron clouds of Fe ions become obvious gradually with the evolution steps. After 100 steps, almost no change can be seen, while after 500 steps, the change has been obvious. To give a better illustration, Fig. 2 shows the maximum value of the charge density change as a function of the evolution steps. The charge density change reaches its maximum after about 800 steps, and the corresponding time is $1.6 \mathrm{~h} /$ $\mathrm{eV}$. It is the time from zero to the maximum. The time of one period should be $4 \times 1.6 \hbar / \mathrm{eV}$, so the frequency is about $160 \mathrm{meV}$. The frequency given in this way is not very accurate and may have an error as large as $\pm 20 \%$, but the frequency is consistent with the authors' guess, indicating that it can be the electron-pairing medium in HTS. Calculations with $2 \times 2 \times 1$ and $6 \times 6 \times 2 k$-point settings give consistent results.

\subsection{FeSe Sheet [27]}

Figure 3 shows the results of FeSe sheet. The $k$-point setting is $2 \times 2 \times 1$ and $3 p^{6} d^{6} 4 s^{2}$ of Fe and $4 s^{2} p^{4}$ of Se are taken as

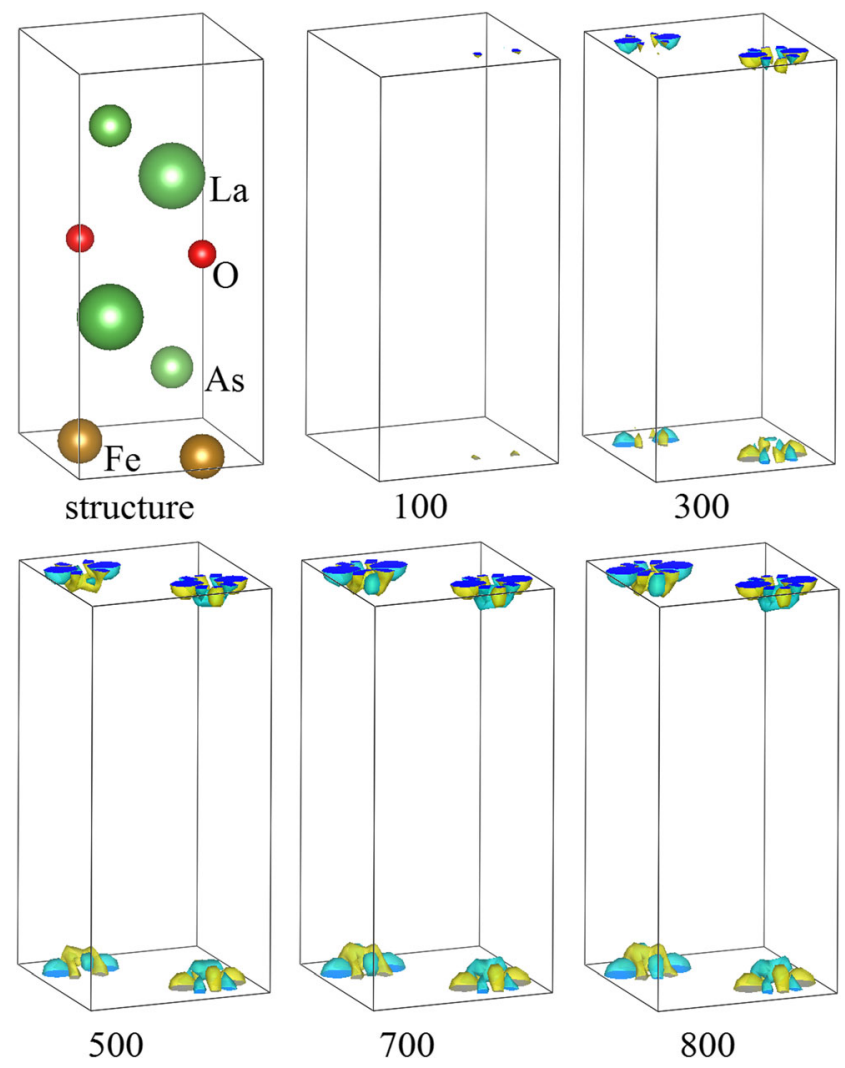

Fig. 1 Crystal structure and the charge density evolution of $\mathrm{La}_{2} \mathrm{Fe}_{2} \mathrm{As}_{2} \mathrm{O}_{2}$. Plots were generated using VESTA [26] (the same below). The isosurface is $0.02 \mathrm{e} / \mathrm{Bohr}^{3}$. The yellow color represents an increase of the charge density, while the blue a decrease (the same below) 


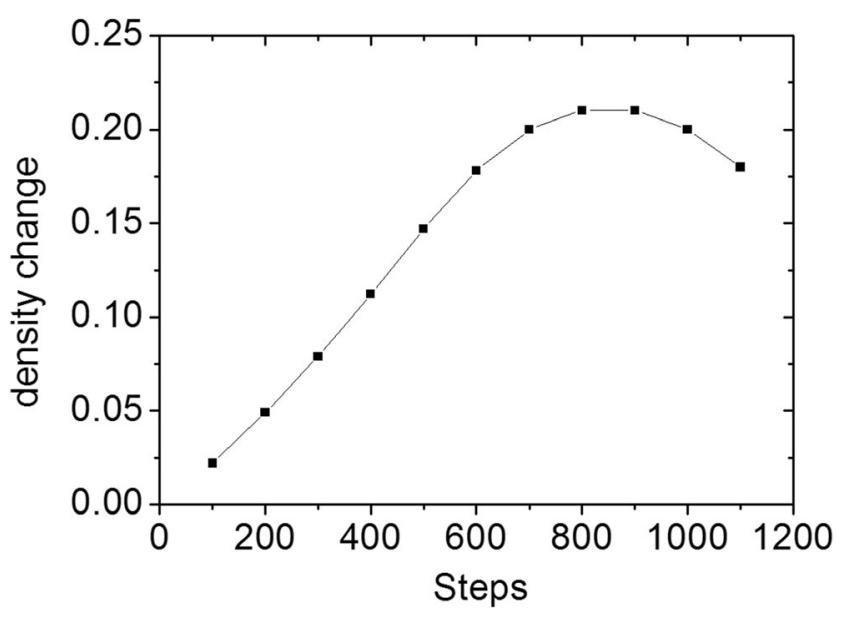

Fig. 2 The relationship between the maximum value of charge density change and the evolution steps

valence electrons. The real space spacing is $0.35 \mathrm{Bohr}$. After 8000 steps, the charge densities are recorded. The change of the electron clouds reaches its maximum after about 650 steps and the frequency is about $190 \mathrm{meV}$. Calculation with a $6 \times$ $6 \times 1 k$-point setting gives consistent results.

\section{$3.3\left(\mathrm{HgBa}_{2} \mathrm{Ca}_{2} \mathrm{Cu}_{3} \mathrm{O}_{8}\right)_{2}$}

The $k$-point setting is $2 \times 2 \times 1$ and $5 \mathrm{p}^{6} \mathrm{~d}^{10} 6 \mathrm{~s}^{2}$ of $\mathrm{Hg}, 5 \mathrm{p}^{6} 6 \mathrm{~s}^{2}$ of $\mathrm{Ba}, 3 \mathrm{p}^{6} 4 \mathrm{~s}^{2}$ of Ca, $3 \mathrm{p}^{6} \mathrm{~d}^{10} 4 \mathrm{~s}^{1}$ of $\mathrm{Cu}$ and $2 \mathrm{~s}^{2} \mathrm{p}^{4}$ of $\mathrm{O}$ are taken as valence electrons. The real space spacing is $0.4 \mathrm{Bohr}$. Figure 4 shows the results excited by a slight change in the position of an $\mathrm{O}$ atom. The original coordinate of the $\mathrm{O}$ ion in the copper oxide plane is $(0.75,0.25,0.0)$, and modified to $(0.8,0.3,0.0)$, which is equivalent to a change of about $0.4 \AA$ along the [110] direction. The ground state was calculated. Then modify the coordinate back to $(0.75,0.25,0.0)$ and conduct TDDFT calculations. After 14000 steps, charge densities were recorded. The change of the electron cloud of the $\mathrm{Cu}$ ion indicated by the arrow reaches its maximum after about 500 steps. The frequency is approximately $250 \mathrm{meV}$.

The characteristic frequencies are $160 \mathrm{meV}, 190 \mathrm{meV}$, and $250 \mathrm{meV}$, respectively, for $\mathrm{La}_{2} \mathrm{Fe}_{2} \mathrm{As}_{2} \mathrm{O}_{2}$, FeSe sheet, and $\mathrm{HgBa}_{2} \mathrm{Ca}_{2} \mathrm{Cu}_{3} \mathrm{O}_{8}$. The results are unexpected, because the general view is that the change of the electron density is very quick and the frequency is much higher than the lattice vibration. The frequencies the author obtained are close to that of the lattice vibration, indicating it can be excited by free electrons. So, the change of the electron clouds of transition metal ions can be the electron-pairing medium. W. A. Little et al. [28] gave similar results, but the frequencies are too high. Whether it can be excited by free electrons should be justified.

We also studied other systems, such as $\mathrm{Fe}_{2} \mathrm{KSe}_{2}$ [29] and $\mathrm{CaCuO}_{2}$ [30]. Similar results were obtained. It is worth noting that the change of the electron clouds of transition metal ions is very complicated. For the same superconductor, there may

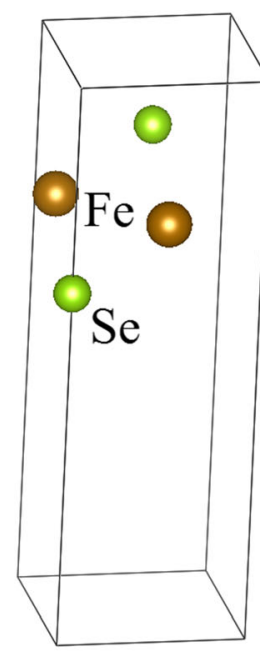

structure

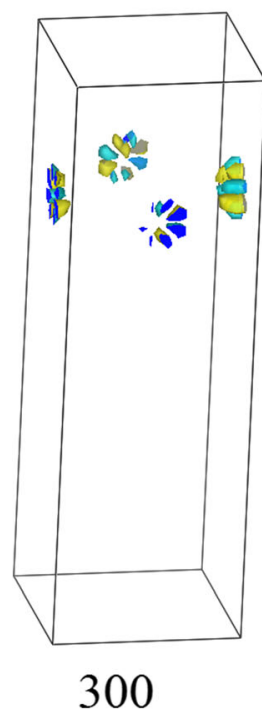

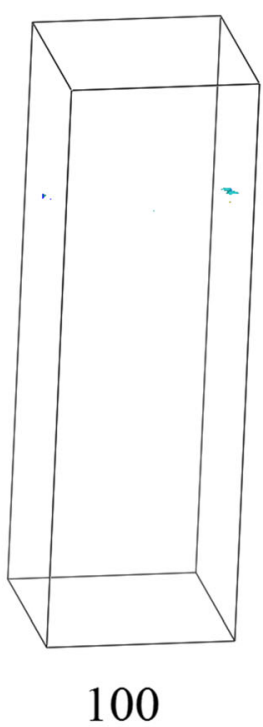
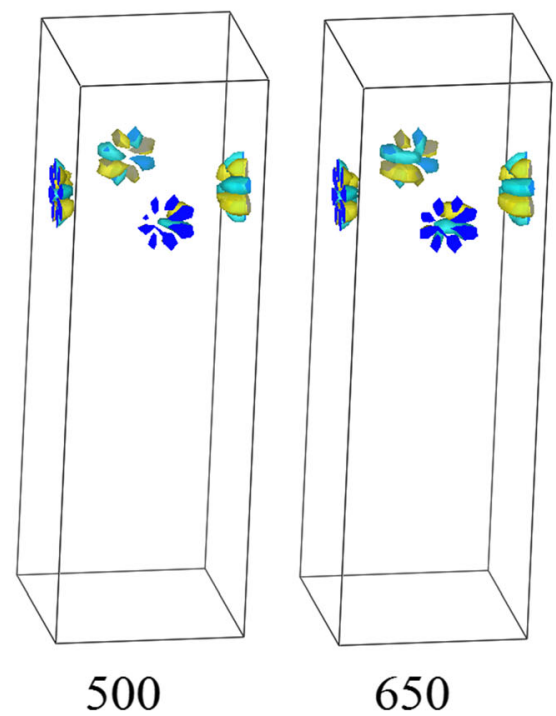

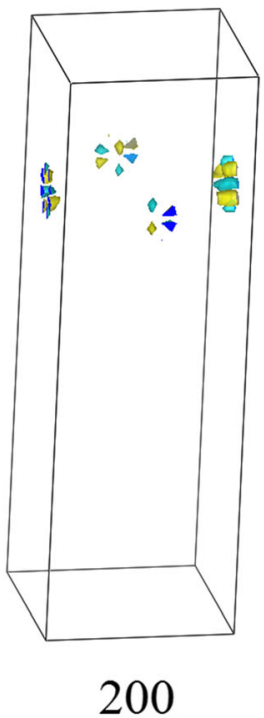

Fig. 3 Crystal structure and the charge density evolution of FeSe sheet. The excitation is the same as above. The isosurface is 0.015

be different modes, corresponding to different frequencies. For the same mode, the frequency is the same. Changing the parameters of the applied electric field may lead to different modes or may not affect the results. Why do the electron clouds of transition metal ions change this way? The main reason is that the $3 d$ shell of transition metal ions is not fully filled. The electron clouds possess no spherical symmetry, and easy to change under electric fields.

\section{Summary}

Real-time evolution of the electron clouds of transition metal ions in unconventional HTS was calculated by the TDDFT method. The frequencies of evolution of electron clouds match well with the frequencies of the lattice vibrations in 

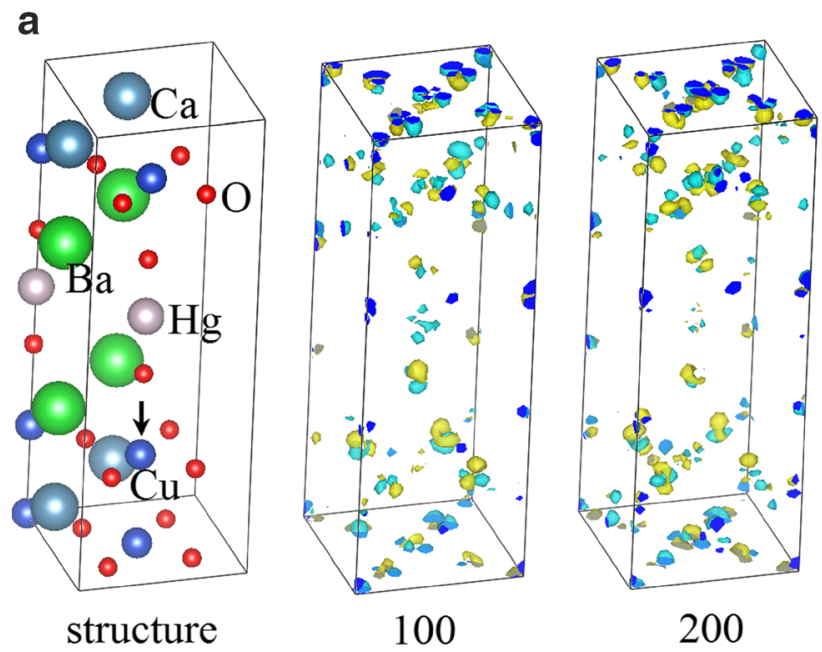

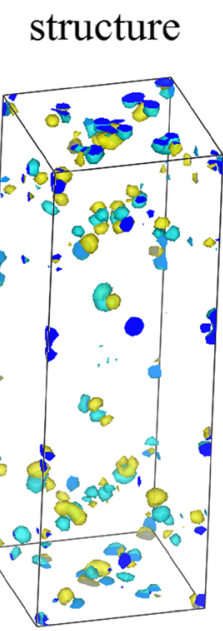

300
100

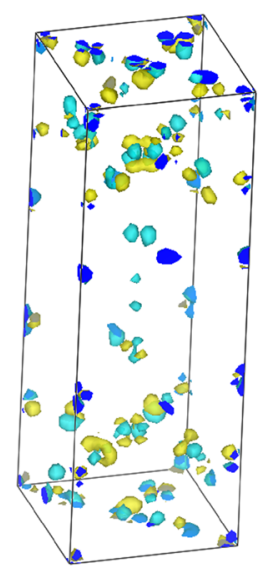

400

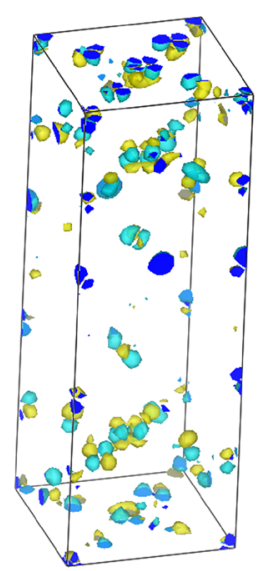

500 b
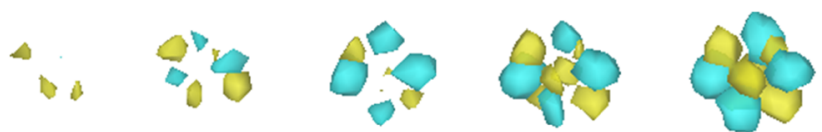

Fig. 4 a Crystal structure and evolution of the charge density of $\left(\mathrm{HgBa}_{2} \mathrm{Ca}_{2} \mathrm{Cu}_{3} \mathrm{O}_{8}\right)$ excited by changing an $\mathrm{O}$ atom. b Enlarged patterns of the charge density change of the $\mathrm{Cu}^{2+}$ ion indicated by the arrow. The isosurface is 0.05

conventional HTS at high pressures. Though the frequencies obtained by this method are not accurate, it can give a significant evidence that the change of electron clouds can be the electron-pairing medium in HTS.

Acknowledgements The author sincerely thanks Xu Zuo, Xinjie Zhao, Feng Lu, Hong Dong, Jian Zhou and Yu Bai for useful discussions.

Funding Information This work was supported by project funded by the China Postdoctoral Science Foundation (2018M640245), project funded by the Hebei Province Postdoctoral Science Foundation (B2018003013), the Natural Science Foundation of Hebei, China (Grant No. F2017208031), the Natural Science Foundation of Nation, China (Grant No. 51674096), and the Fundamental Research Funds for the Central Universities, Nankai University (Grant No. 63191740).
Open Access This article is distributed under the terms of the Creative Commons Attribution 4.0 International License (http:// creativecommons.org/licenses/by/4.0/), which permits unrestricted use, distribution, and reproduction in any medium, provided you give appropriate credit to the original author(s) and the source, provide a link to the Creative Commons license, and indicate if changes were made.

\section{References}

1. Bednorz, J.G., Müller, K.A.: Possible high $T_{\mathrm{C}}$ superconductivity in the Ba-La-Cu-O system. Zeitschrift für Physik B. 64, 189-193 (1986)

2. Wu, M.K., Ashburn, J.R., Torng, C.J., Hor, P.H., Meng, R.L., Gao, L., Huang, Z.J., Wang, Y.Q., Chu, C.W.: Superconductivity at $93 \mathrm{~K}$ in a new mixed-phase $\mathrm{Y}-\mathrm{Ba}-\mathrm{Cu}-\mathrm{O}$ compound system at ambient pressure. Phys. Rev. Lett. 58, 908-910 (1987)

3. Kamihara, Y., Hiramatsu, H., Hirano, M., Kawamura, R., Yanagi, H., Kamiya, T., Hosono, H.: Iron-based layered superconductor: LaOFeP. J. Am. Chem. Soc. 128(31), 10012-10013 (2006)

4. Kamihara, Y., Watanabe, T., Hirano, M., Hosono, H.: Iron-based layered superconductor $\mathrm{La}\left[\mathrm{O}_{1-\mathrm{x}} \mathrm{F}_{\mathrm{x}}\right] \mathrm{FeAs}(x=0.05-0.12)$ with $T_{\mathrm{c}}=$ 26 K. J. Am. Chem. Soc. 130, 3296-3297 (2008)

5. Bardeen, J., Cooper, L.N., Schrieffer, J.R.: Microscopic theory of superconductivity. Phys. Rev. 106, 162-164 (1957)

6. Bardeen, J., Cooper, L.N., Schrieffer, J.R.: Theory of superconductivity. Phys. Rev. 108, 1175-1204 (1957)

7. Anderson, P.W.: The resonating valence bond state in $\mathrm{La}_{2} \mathrm{CuO}_{4}$ and superconductivity. Science. 235, 1196-1198 (1987)

8. Anderson, P.W.: Twenty years of talking past each other: the theory of high $\mathrm{T}_{\mathrm{C}}$. Physica C. 460, 3-6 (2007)

9. Monthoux, P., Balatsky, A., Pines, D.: Weak-coupling theory of high-temperature superconductivity in the antiferromagnetically correlated copper oxides. Phys. Rev. B. 46, 14803-14817 (1992)

10. Chakravarty, S., Sudbø, A., Anderson, P.W., Strong, S.: Interlayer tunneling and gap anisotropy in high-temperature superconductors. Science. 261, 337-340 (1993)

11. Anderson, P.W.: Is there glue in cuprate superconductors? Science. 22, 1705-1707 (2007)

12. Kontani, H., Onari, S.: Orbital-fluctuation-mediated superconductivity in iron Pnictides: analysis of the five-orbital HubbardHolstein model. Phys. Rev. Lett. 104, 157001 (2010)

13. Saito, T., Onari, S., Kontani, H.: Orbital fluctuation theory in iron pnictides: effects of As-Fe-As bond angle, isotope substitution, and $\mathrm{Z}^{2}$-orbital pocket on superconductivity. Phys. Rev. B. 82, 144510 (2010)

14. Stanescu, T.D., Galitski, V., Sarma, S.D.: Orbital fluctuation mechanism for superconductivity in iron-based compounds. Phys. Rev. B. 78, 195114 (2008)

15. Koga, A., Kawakami, N., Rice, T.M., Sigrist, M.: Spin, charge, and orbital fluctuations in a multiorbital Mott insulator. Phys. Rev. B. 72, 045128 (2005)

16. Poncé, S., Margine, E.R., Verdi, C., Giustino, F.: EPW: Electronphonon coupling, transport and superconducting properties using maximally localized Wannier functions. Comput. Phys. Commun. 209, 116-133 (2016)

17. Durajski, A.P., Szczęśniak, R.: First-principles study of superconducting hydrogen sulfide at pressure up to $500 \mathrm{Gpa}$. Sci. Rep. 7, 4473 (2017)

18. Zhou, T.G. Rotation of transition metal ions under electric fields: possible new superconducting electron pairing mechanism. http:// vixra.org/abs/1804.0338 (2018) 
19. Castro, A., Marques, M.A.L., Alonso, J.A., Rubio, A.: Optical properties of nanostructures from time-dependent density functional theory. J. Comp. Theoret. Nanoscience. 1, 231-255 (2004)

20. Marques, M.A.L., Gross, E.K.U.: Time-dependent density functional theory. Annu. Rev. Phys. Chem. 55, 427-455 (2004)

21. Andrade, X., Strubbe, D.A., De Giovannini, U., Larsen, A.H., Oliveira, M.J.T., Alberdi-Rodriguez, J., Varas, A., Theophilou, I., Helbig, N., Verstraete, M., Stella, L., Nogueira, F., Aspuru-Guzik, A., Castro, A., Marques, M.A.L., Rubio, A.: Real-space grids and the Octopus code as tools for the development of new simulation approaches for electronic systems. Phys. Chem. Chem. Phys. 17, 31371-31396 (2015)

22. Marques, M.A.L., Castro, A., Bertsch, G.F., Rubio, A.: Octopus: a first-principles tool for excited electron-ion dynamics. Comput. Phys. Commun. 151, 60-78 (2003)

23. Agapito, L.A., Curtarolo, S., Nardelli, M.B.: Reformulation of DFT $+\mathrm{U}$ as a pseudohybrid Hubbard density functional for accelerated materials discovery. Phys. Rev. X. 5(1), 011006 (2015)

24. Tancogne-Dejean, N., Oliveira, M.J.T., Rubio, A.: Self-consistent $\mathrm{DFT}+\mathrm{U}$ method for real-space time-dependent density functional theory calculations. Phys. Rev. B. 96, 245133 (2017)

25. Vanderbilt, D.: Optimally smooth norm-conserving pseudopotentials. Phys. Rev. B. 32, 8412-8115 (1985)
26. Momma, K., Izumi, F.: VESTA: a three-dimensional visualization system for electronic and structural analysis. J. Appl. Crystallogr. 41, 653-658 (2008)

27. Ge, J.F., Liu, Z.L., Liu, C.H., Gao, C.L., Qian, D., Xue, Q.K., Liu, Y., Jia, J.F.: Superconductivity above $100 \mathrm{~K}$ in single-layer FeSe films on doped $\mathrm{SrTiO}_{3}$. Nat. Mater. 14, 285-289 (2015)

28. Little, W.A., Holcomb, M.J., Ghiringhelli, G., Braicovich, L., Dallera, C., Piazzalunga, A., Tagliaferri, A., Brookes, N.B.: A determination of the pairing interaction in the high $T_{\mathrm{c}}$ cuprate superconductor $\mathrm{Tl}_{2} \mathrm{Ba}_{2} \mathrm{CaCu}_{2} \mathrm{O}_{8}$ (T12212). Physica C. 460-462, 40-43 (2007)

29. Ricci, A., Poccia, N., Joseph, B., Arrighetti, G., Barba, L., Plaisier, J., Campi, G., Mizuguchi, Y., Takeya, H., Takano, Y., Saini, N.L., Bianconi, A.: Intrinsic phase separation in superconducting $\mathrm{K}_{0.8} \mathrm{Fe}_{1.6} \mathrm{Se}_{2}\left(T_{\mathrm{c}}=31.8 \mathrm{~K}\right)$ single crystals. Supercond. Sci. Technol. 24, 082002 (2011)

30. Siegrist, T., Roth, R.S., Zahurak, S.M., Murphy, D.W.: The parent structure of the layered high-temperature superconductors. Nature. 334, 231-232 (1988)

Publisher's Note Springer Nature remains neutral with regard to jurisdictional claims in published maps and institutional affiliations. 